\title{
Molecular cloning, characterization and tissue distribution of six splice variants of activin type IIA receptor (ActRIIA) from grass carp (Ctenopharyngodon idellus)
}

\author{
Chao Chen, Chunlei Song, Xinyan Wang and Hong Zhou* \\ Key Laboratory for Neuroinformation of Ministry of Education, School of Life Science \\ and Technology, University of Electronic Science and Technology of China, \\ Chengdu, 610054, People's Republic of China
}

(Received 25 September 2009, accepted 12 November 2009)

\begin{abstract}
Activin type II receptor (ActRII) is crucial for the assembly of the ligand/receptor complex and the activation of downstream cascades in activin signaling pathway. In this study, we identified six variants of grass carp ActRIIA which can be generated through three alternative splicing events (defined as AS1, AS2 and AS3). AS1 induces a spliced segment encoding 14 amino acids located in the external juxtamembrane region of the receptor. However, both AS2 and AS3 occur at the same cleavage site of kinase domain and induce a premature termination codon. Indeed, AS2 inserts a fragment of $79 \mathrm{bp}$ while AS3 generates a new 3' terminal of cDNA with poly(A) signals. The full length cDNA of the shortest variant was shown to be $2001 \mathrm{bp}$ encoding 514 amino acids with sequence identity of $79-95 \%$ to counterparts in other species. Homology modeling studies showed grass carp ActRIIA exhibits a characteristic three-finger toxin fold in the extracellular domain and a conserved bilobal architecture in the intracellular kinase domain. Semi-quantitative RT-PCR analysis revealed that six variants showed different expression patterns in selected tissues of grass carp. This study will be helpful for a better understanding of the physiological role of activin signaling in lower vertebrates.
\end{abstract}

Key words: activin type IIA receptor (ActRIIA), alternative splicing, grass carp, tissue distribution

\section{INTRODUCTION}

Activin belongs to the transforming growth factor- $\beta$ (TGF- $\beta$ ) superfamily, which comprises a number of structurally related and multifunctional growth factors, including TGF- $\beta$, activin/inhibin, bone morphogenetic protein (BMP), growth and differentiation factor, as well as nodal (Shi and Massague, 2003). Activin was initially characterized as a stimulator of follicle-stimulating hormone (FSH) secretion in the anterior pituitary gland (Ling et al., 1986; Vale et al., 1986). Subsequently, activin has been shown to play crucial roles in many developmental and physiological events including cell proliferation and differentiation (Mizuguchi et al., 1993; Wang et al., 1996; Wang et al., 2007), inflammatory responses (Jones et al., 2007; Robson et al., 2008), and embryonic stem cell renewal (Xiao et al., 2006).

Edited by Yasuyoshi Nishida

* Corresponding author. E-mail: zhouhongzh@uestc.edu.cn
It is well known that activin exerts its multiple biological functions via binding to its specific membrane receptors (Shi and Massague, 2003). There are two types of activin receptors, namely type I and type II receptors with protein serine/threonine kinase activity (Attisano et al., 1993; Donaldson et al., 1992). Upon ligand binding, type II receptors (ActRIIA and ActRIIB) recruit and phosphorylate type I receptors at the serine residues within the highly conserved GS domain (a glycine/serine-rich region). The activated type I receptors in turn phosphorylate receptor-regulated Smads (R-Smad, Smad2 and Smad3), allowing R-Smad to form a heterocomplex with co-mediator Smad (Smad4), and the Smad complex is translocated into the nucleus to regulate target gene transcription (Greenwald et al., 1999; Shi and Massague, 2003). Therefore, type II receptors are responsible for ligand recognition and binding, as well as signal transduction in the activin signaling pathway. In mammals, only five type II receptors have been identified, whereas over 35 TGF- $\beta$ ligands have been discovered (Chang et al., 2002). Actu- 
ally, different ligands in TGF- $\beta$ superfamily may signal through the same receptor, e.g. both activin and subset of BMP (BMP6/7) signal via ActRIIA (Lavery et al., 2008) and drive the formation of distinct receptor complexes, thus initiating different cellular responses. Notably, mouse ActRIIA (Shoji et al., 1998) and ActRIIB (Attisano et al., 1992) have been shown to undergo alternative splicing, which has been implicated to regulate the relative ligand affinities and subsequent signal transduction pathways, indicating that the generation of diverse isoforms of activin receptor through alternative splicing may provide a molecular basis corresponding to the complicated biology of activin and related factors.

In fish, ActRIIA sequence has been cloned from zebrafish (Nagaso et al., 1999; DiMuccio et al., 2005). The expression patterns of ActRIIA in embryonic development have also been investigated in zebrafish (Nagaso et al., 1999). Grass carp (Ctenopharyngodon idellus) is an important aquaculture species in Asian countries. In this study, we cloned a new member of the fish ActRIIA family, which is present in six distinct forms generated by three alternative splicing events in this species. These receptor variants differ from each other in the external juxtamembrane region and kinase domain, and express differentially in all tissues examined. The existence of alternatively spliced ActRIIA isoforms provides the new insight for understanding the functions of activin system in lower vertebrates.

\section{MATERIALS AND METHODS}

Animals, tissue sampling and total RNA extraction One-year-old grass carp (Ctenopharyngodon idellus) (weighing 500-1000 g) were purchased from Chengdu Tongwei Aquatic Science and Technology Company, China, and were routinely fed once daily with natural temperature and photoperiod for 2 weeks prior to use. Grass carp were anaesthetized and dissected aseptically to collect the following tissues: pituitary, brain, thymus, heart, head kidney, kidney, spleen, muscle and gonad. Approximate $50 \mathrm{mg}$ of each tissue was used for total RNA extraction with TRIzol Reagent (Invitrogen, USA). All RNA samples were treated with DNase I (Invitrogen, USA) to remove any contaminating DNA and the DNAse was subsequently inactivated by EDTA and heated at $65^{\circ} \mathrm{C}$ before reverse transcription.

\section{Isolation of the full-length cDNA of ActRIIA}

About $5 \mu \mathrm{g}$ of total RNA from grass carp heart were reverse transcribed with oligo $(\mathrm{dT})_{18}$ using the Superscript II reverse transcription kit (Invitrogen, USA). Degenerate PCR was performed to obtain the partial cDNA of ActRIIA with the primer pair DF and DR (Table 1), which were designed based on multiple alignment of the ActRIIA nucleotide sequences of zebrafish, chicken, frog, human, mouse and cattle. The cycling protocol for degenerate $\mathrm{PCR}$ was one cycle of $94^{\circ} \mathrm{C}$ for $5 \mathrm{~min}, 35$ cycles of $94^{\circ} \mathrm{C}$ for $30 \mathrm{~s}, 57^{\circ} \mathrm{C}$ for $30 \mathrm{~s}, 72^{\circ} \mathrm{C}$ for $1 \mathrm{~min}$, followed by one cycle of $72^{\circ} \mathrm{C}$ for $10 \mathrm{~min}$. PCR products were separated on a $1.0 \%$ agarose gel, stained with ethidium bromide solution and visualized by the Universal HOOD II-S.N 76s Imaging-system (Bio-Rad, USA). Target PCR fragments were purified using the ToPure ${ }^{\mathrm{TM}}$ Gel DNA Extraction Kit (Genetech, China) and subcloned into pGM-T vector (TaKaRa, Japan). Selected clones were sequenced using the ABI $3730 \mathrm{XL}$ sequencer (Applied Biosystems, USA).

ActRIIA-specific primers (Table 1) were designed based on the partial cDNA of ActRIIA to isolate the full-length cDNA by Rapid Amplification of cDNA Ends (RACE) by using the GeneRacer Kit (Invitrogen, USA). PCR conditions were one cycle of $94^{\circ} \mathrm{C}$ for $5 \mathrm{~min}$, followed by 35 cycles of $94^{\circ} \mathrm{C}$ for $30 \mathrm{~s}, 60^{\circ} \mathrm{C}$ (for $5^{\prime}$-RACE) $/ 63^{\circ} \mathrm{C}$ (for $3^{\prime}$ RACE) for $30 \mathrm{~s}, 72^{\circ} \mathrm{C}$ for $1 \mathrm{~min}$, with a final extension at $72^{\circ} \mathrm{C}$ for $10 \mathrm{~min}$. PCR products generated by 5' and $3^{\prime}$ RACE were subcloned and sequenced as described above. These cDNA fragments were assembled on the basis of overlapped sequences, and then ActRIIA-specific primers (5UTR, CSP2 and TRCF, Table 1) located in 5' and 3' untranslated regions (UTR) were designed to perform the end-to-end PCR by using high fidelity Pfu polymerase (Stratagene, USA) to validate the full-length cDNA of ActRIIA.

Sequence analysis of grass carp ActRIIA Identification of open reading frames and translation were conducted with the online ORFfinder on the server of the National Center for Biotechnology Information (NCBI) (http://www.ncbi.nlm.nih.gov). Similarity searches of the cDNA sequences and deduced protein sequences were implemented using the Basic Local Alignment Search Tool (BLAST) (Altschul et al., 1990) at the NCBI. The signal peptide was predicted by using the Hidden Markov Model approach supplied in SignalP 3.0 (http://www. cbs.dtu.dk/services/SignalP-3.0/) (Bendtsen et al., 2004). Transmembrane spanning prediction was achieved by using the Transmembrane Hidden Markov Model program (TMHMM) (http://www.cbs.dtu.dk/services/TMHMM -2.0/). Analysis on characteristic motifs and domains was conducted by using Motifscan (http://myhits.isb-sib.ch/cgibin/motif_scan) (Falquet et al., 2002). Alignment of deduced amino acid sequences was performed by using DNAMAN software (Lynnon Biosoft, Canada). In order to understand the secondary structures of the extracellular domain and kinase domain of grass carp ActRIIA, secondary structure-based alignment was conducted with the known structures of mouse ActRII and human ActRIIB (Greenwald et al., 1999; Han et al., 2007). Furthermore, modeling of tertiary structure for grass carp ActRIIA was achieved by using the automated compara- 
Table 1. Primers used in RT-PCR

\begin{tabular}{|c|c|c|}
\hline Primer name & Sequence (5'-3') & Use \\
\hline $\mathrm{DF}$ & TGGCTKGATGATRTCAACTG & Isolation of partial cDNA fragment \\
\hline $\mathrm{DR}$ & TCAGCWATRCARGCTGTCAG & Isolation of partial cDNA fragment \\
\hline Oligo $(\mathrm{dT})_{1 \mathrm{~S}}$ & TTTTTTTTTTTTTTTTTT & Synthesis of the first-strand cDNA for 5'RACE \\
\hline $5 \mathrm{P} 1$ & TCTTCACTGCCACATAGTCATTG & First PCR of 5'RACE \\
\hline $5 \mathrm{P} 2$ & GTAGAAGAACTTCTCGTTGCAC & Nested PCR of 5'RACE \\
\hline AAP & GGCCACGCGTCGACTAGTACGGGIIGGGIIGGGIIG & First PCR of 5'RACE \\
\hline AUAP & GGCCACGCGTCGACTAGTAC & Nested PCR of 5'RACE \\
\hline $\mathrm{AP}$ & $\begin{array}{l}\text { GCTGTCAACGATACGCTACGTAACGGCATGACAG } \\
\text { TGTTTTTTTTTTTTTTTTTT }\end{array}$ & Synthesis of the first-strand cDNA for 3'RACE \\
\hline $3 \mathrm{P} 1$ & CTGGATGTATCGGCATCACAAG & First PCR of 3'RACE \\
\hline $3 \mathrm{P} 2$ & CATTGAGTTGTGGCTCATCACG & Nested PCR of 3'RACE \\
\hline 3AP1 & GCTGTCAACGATACGCTACGTAACG & First PCR of 3'RACE \\
\hline 3AP2 & CGCTACGTAACGGCATGACAGTG & Nested PCR of 3'RACE \\
\hline 5UTR & ATGCAGAGACTCTTGGATTTTGTAG & End-to-end PCR \\
\hline CSP & TAGACTGGACTCCTTAGGGGGAT & End-to-end PCR \\
\hline TRCF & GTTACCAACTCAATCACACTGTC & End-to-end PCR, Tissue distribution of variant 5 and 6 \\
\hline $\mathrm{I} 42 \mathrm{~F}$ & TCAGCGACACAAACAGCAAAGTC & Tissue distribution of variant 2 and 4 \\
\hline $\mathrm{S} 42 \mathrm{~F}$ & ATCCCGCCAGTTCAGACAACATC & Tissue distribution of variant 1 and 3 \\
\hline I79R & CATCTGTTCAGATCAGGAAGTC & Tissue distribution of variant 3 and 4 \\
\hline S79R & CTCGTCAACTGGACCATCAGAG & Tissue distribution of variant 1 and 2 \\
\hline $\mathrm{P} 1$ & CAACGAGAAGTTCTTCTACAACC & Tissue distribution of variant 5 and 6 \\
\hline$\beta$-actin $\mathrm{F}$ & TTGGTGACGAGGCTCAGAGCA & Internal control \\
\hline$\beta$-actin $\mathrm{R}$ & CACCATCACCAGAGTCCATCAC & Internal control \\
\hline
\end{tabular}

tive protein modeling server SWISS-MODEL (http:// swissmodel.expasy.org) with the first approach mode. In this case, the crystal structures of the extracellular ligand-binding domain of mouse ActRII (PDB 1bteB) (Greenwald et al., 1999) and human ActRIIB kinase domain (PDB 2qluA) (Han et al., 2007) were utilized as the templates to create the ligand-binding domain and the intracellular kinase domain of grass carp ActRIIA, respectively. The three-dimensional (3D) models were visualized with Molsoft ICM software (Molsoft L.L.C., USA). The serine/threonine protein kinase active-site was identified by Motifscan and ScanProsite (http:// www.expasy.org/tools/scanprosite/).

Phylogenetic tree construction The phylogenetic tree was constructed with the Neighbor-Joining method by using a MEGA 3.1 software (Kumar et al., 2004), with the bootstrapping of 1,000 repetitions. All type II serine/ threonine kinase receptors of TGF- $\beta$ family reported to date were searched and downloaded from the nonredundant protein sequences database in NCBI. The following shows the accession numbers of these proteins: zebrafish ActRIIA (NP_001103748), frog ActRII (NP_001084061), chicken ActRIIA (NP_990698), bovine
ActRIIA (NP_776652), sheep ActRIIA (NP_001009293), human ActRIIA (AAH67417), mouse ActRIIA (AAI38824), rat ActRIIA (NP_113759), grass carp ActRIIB (ACI23559), zebrafish ActRIIB (NP_571285), Atlantic salmon ActRIIB (ABK54368), frog ActRIIB (NP_001084049), chicken ActRIIB (NP_989648), bovine ActRIIB (NP_776920), pig ActRIIB (NP_001005350), human ActRIIB (AAC64515), mouse ActRIIB (NP_031423), rat ActRIIB (NP_113742), zebrafish BMPRIIA (NP 001034906), zebrafish BMPRIIB (CAQ13984), frog BMPRII (NP_001081659), chicken BMPRII (NP_001001465), human BMPRII (AAH52985), mouse BMPRII (NP_031587), rat BMPRII (EDL98946), zebrafish TGFBRII (NP_878275), chicken TGFBRII (NP_990759), American mink TGFBRII (A44225), human TGFBRII (AAT70724), rat TGFBRII (NP_112394), mouse TGFBRII (AAB30100).

Tissue distribution patterns of grass carp ActRIIA mRNA RT-PCR was conducted to explore the spatial patterns of the alternative spliced transcripts of ActRIIA in grass carp. Total RNA was isolated from pituitary, brain, thymus, heart, head kidney, kidney, spleen, muscle, gonad, and then reverse transcribed using M-MLV transcriptase (Promega, USA) with oligo(dT) 18 . Variant- 
specific primers were designed and used to detect the expression of individual transcript variants (Fig. 5A). To optimize the cycle number for semi-quantitative analysis, PCR amplification profiles were analyzed using a cDNA pool of various tissues as the template. Amplification of $\beta$-actin was used as the internal control. PCR was done for 30 to 40 cycles, with annealing temperatures from $60^{\circ} \mathrm{C}$ to $62^{\circ} \mathrm{C}$ depending on the target genes. PCR products were separated on the agarose gel, stained and visualized as described above.

A
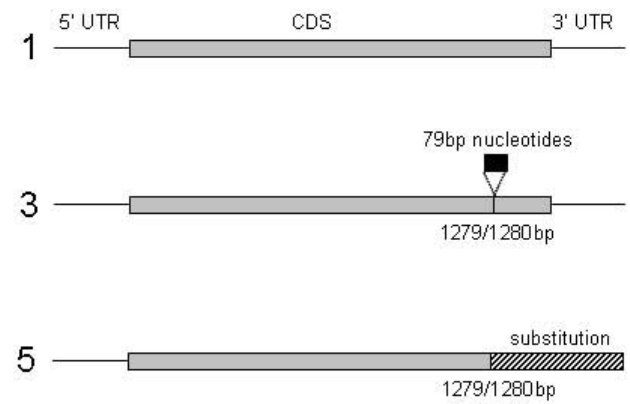

\section{RESULTS}

Molecular cloning and nucleotide sequence analysis of grass carp ActRIIA Two cDNA fragments of $821 \mathrm{bp}$ and $779 \mathrm{bp}$ were obtained from degenerate PCR and they share $88 \%$ and $92 \%$ nucleotide identity with zebrafish ActRIIA, respectively. Multiple alignment analysis reveals that the shorter nucleotide sequence is similar to zebrafish homolog, whereas the longer cDNA fragment harbors a $42 \mathrm{bp}$ insert of nucleotide sequence when compared with the shorter one and ActRIIAs from the other species. 5'- and 3'-RACE were conducted to iso-

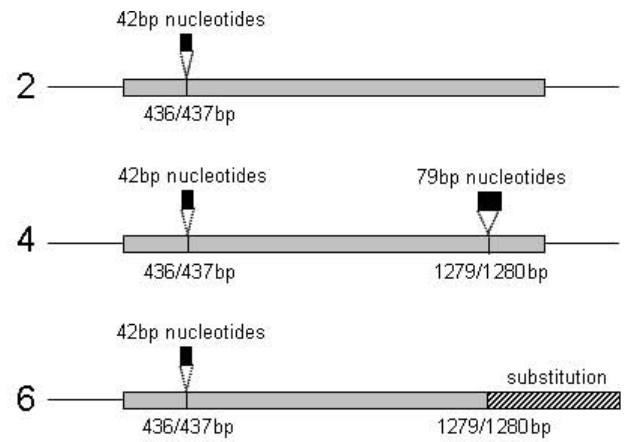

$\mathrm{B}$

GAGGGGAACTTGAMTCAGAGACTCTTGGATTTTGTAGGACAMGACAGAMGATTGAMA 60 ATGGGACCTGCAMCAMGCTGGCGTTTGGAGTGTTCCTCATCTCCTGCTCCTCAGGTGCCATTCTCGGCCGCTCAGAGACACAMGAGTGTGTGTTCTACAMCTACAMCCCCAGTTTGGAG 18O

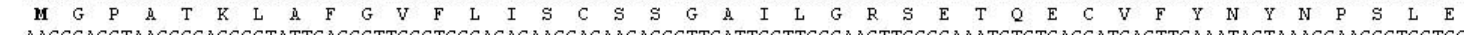

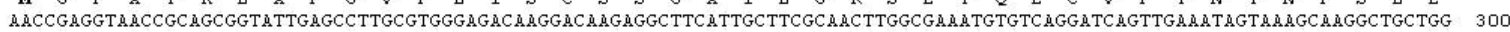

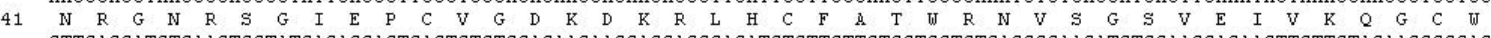

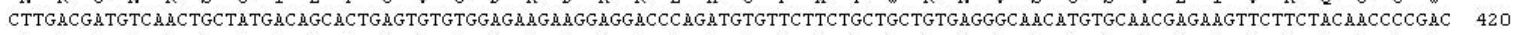

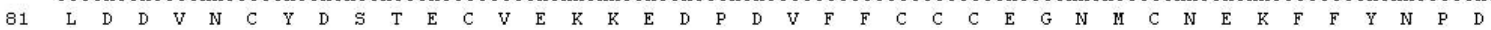

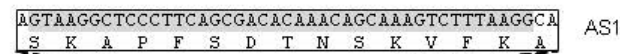

\begin{tabular}{llllllllllllllll}
$\mathrm{S}$ & $\mathrm{K}$ & $\mathbf{A}$ & $\mathrm{P}$ & $\mathrm{F}$ & $\mathrm{S}$ & $\mathrm{D}$ & $\mathrm{T}$ & $\mathrm{N}$ & $\mathrm{S}$ & $\mathrm{K}$ & $\mathrm{V}$ & $\mathrm{F}$ & $\mathrm{K}$ & A \\
\hline
\end{tabular}

ATCCCGCCAGTTCAGMCA A CATCCAMCCCCTTCACCCAGAMGCACCACTGTTCAGCACCCTCCTTTACTCCATCGTGCCCATCATGGGCATCGCTGCCATTGTCCTGCTCTCCTTCTGG 54O

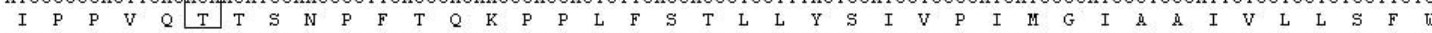
ATGTATCGGCATCACAMGCTAGCGTATCCACCTGTTCTGGTGCCTACCCAGGACCCTGGACCCATGCCGCCGTCTCCCACCCTGGTCCAGMAGCCACTGCAGTTGTTGGMATCAMAGCT 660

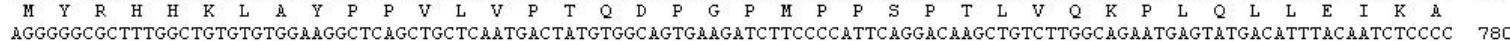
AGGGGGCGCTTTGGCTGTGTGTGGAMGGCTCAGCTGCTCAMTGACTATGTGGCAGTGAMGATCTTCCCCATTCAGGACAAGCTGTCTTGGCAGAMTAGTATGACATTACAATCTCCCC

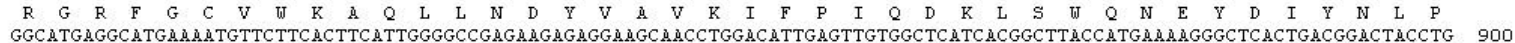

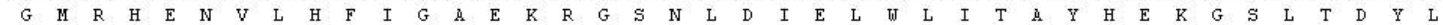
AMGGCCAMCGTAGTGACGTGGAMCGAMCTATGTCACATCGCTCAGACCATGGCTCGAGGACTGGCTTACTTGCACTCTGACTTCCCTGGACACAGMGATGGCCACAMACCGGCCATTGCA 1020

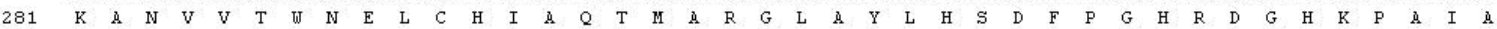
CACAGGGATATTAMGAGCAMGAMTGTGCTGCTGAMGACAMACCTGACAGCCTGTATAGCTGACTTTGGTCTGGCTCTGAGGTTTGAGGCAGGGAMGTCAGCTGGGGACACGCATGGGCAG 1140

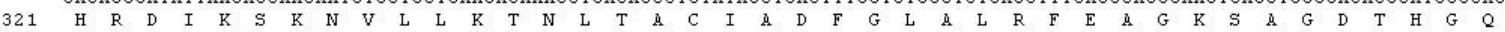
GTGGGAMCAGGCGTTACATGGCTCCGGAGGTGTTGGAGGGGGCCATCAMCTTCCAGCGAGACTCTTTCCTCAGGATAGACATGTATGCAGTCGGGCTGGTGCTTTGGGAGCTGGCGGCC 12 60

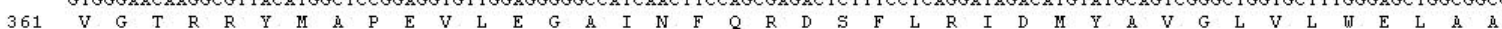
GCTTCCTGACCCAGTTAGMAMACACCCTCAGTGGTTGGMCTTCCTGMTCTGMACMGATGTTTGTTCATTTTTTCTGMAGT AS2

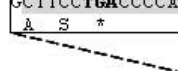

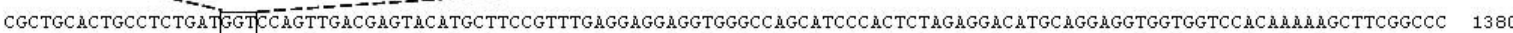

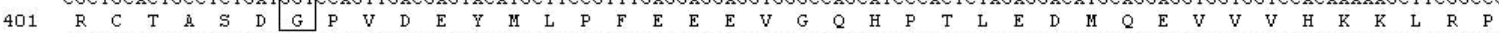

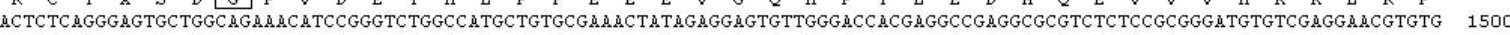

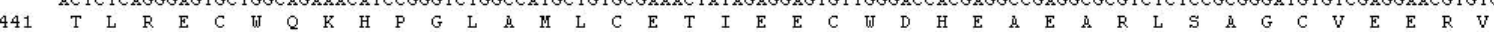

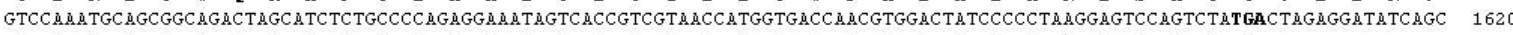
$\begin{array}{lllllllllllllllllllllllllllllllllllll}V & Q & M & Q & R & Q & T & S & I & S & A & P & E & E & I & V & T & V & V & T & M & V & T & N & V & D & Y & P & P & K & E & S & S & L\end{array}$

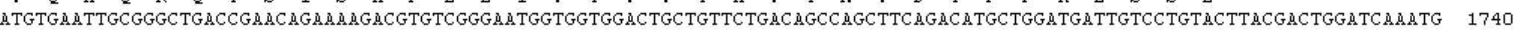

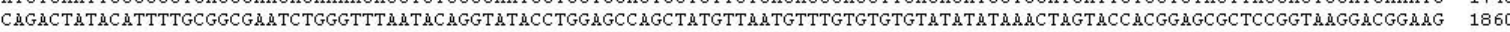
ATTATAMGCCGGCATCAMAMACGTTGTTGTCGTTTCTCTGCTTGGMGÄAMACTTTCCCGTTCAMGGAGTAMACGAGACTCCTCCGCACGGTCATTTCAGTATAMAMAGGGCAMCGTCC 1980

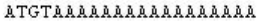

AS3

CGCTGCACTGCCTCTGATGGTATGAMTGCGTGAGCTGCTGTTCCTTATGACCGTTTTGACATCACACTCTACAGCAGGGCTACTCAMTTAGCTTCATTTTTGGGCGGATTATCGAMTTG 1380

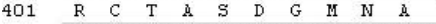

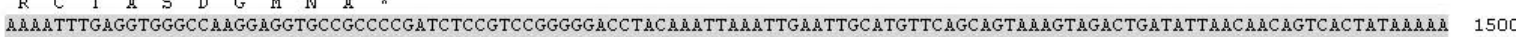

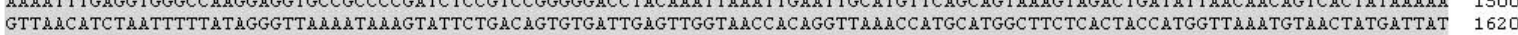
GGTTAMGATTCTATAMACTATATTTGTTTMAMAMCAGTAGTTTAMACGGGTAMAMAMAMAMAMAM

Fig. 1. (A) Schematic representation of six transcript variants of grass carp ActRIIA. The position and length (bp) of alternative splicing were shown. (B) The nucleotide and deduced amino acid sequences of the grass carp ActRIIA with three alternative splicing events. Nucleotides and amino acids are numbered on the right and left, respectively. The start and stop codons are in bold letters. The inserted and substituted fragments are indicated by grey background. The polyadenylation signals are underlined. 
late 5' and 3' flanking regions of ActRIIA, respectively. The 5'-RACE obtained one single fragment of $411 \mathrm{bp}$ which shares $89 \%$ nucleotide identity with zebrafish ActRIIA. However, 3'-RACE yielded three sequences with the size of $1159 \mathrm{bp}, 1238 \mathrm{bp}$ and $847 \mathrm{bp}$. These partial fragments derived from degenerate PCR and RACE were assembled on the basis of overlapped sequences to get the full-length cDNA of grass carp ActRIIA. In this case, three novel alternative splicing events (AS) were observed within grass carp ActRIIA mRNA (Fig. 1). We also performed the end-to-end PCR by using high fidelity Pfu polymerase, followed by cloning and sequencing to exclude the possibility of PCR error and confirm the existence of six transcript forms of grass carp ActRIIA (defined as variant 1-6 (Genbank accession nos.
FJ686113-FJ686118) as shown in Fig. 1A). The cDNA of variant 1 possesses a $60 \mathrm{bp}$ 5' UTR, a $1545 \mathrm{bp}$ open reading frame and a $396 \mathrm{bp}$ 3'UTR, which exhibits the highest similarity with the homologs from the other species. Given that the variant 1 is defined as the standard form of grass carp ActRIIA, the observed three alternative splicing types are: (1) An insert of $42 \mathrm{bp}$ nucleotides at $436 / 437$ bp of variant 1 , which was designated as alternative splicing type 1 (AS1). (2) An insert of $79 \mathrm{bp}$ nucleotides at $1279 / 1280 \mathrm{bp}$ of variant 1 , which was designated as alternative splicing type 2 (AS2). (3) The substitution of 3' terminal of cDNA after 1279 bp by a new nucleotide sequence, which was designated as alternative splicing type 3 (AS3). Notably, AS2 and AS3 occur at the same site in the cDNA of variant 1 (Fig. 1B).

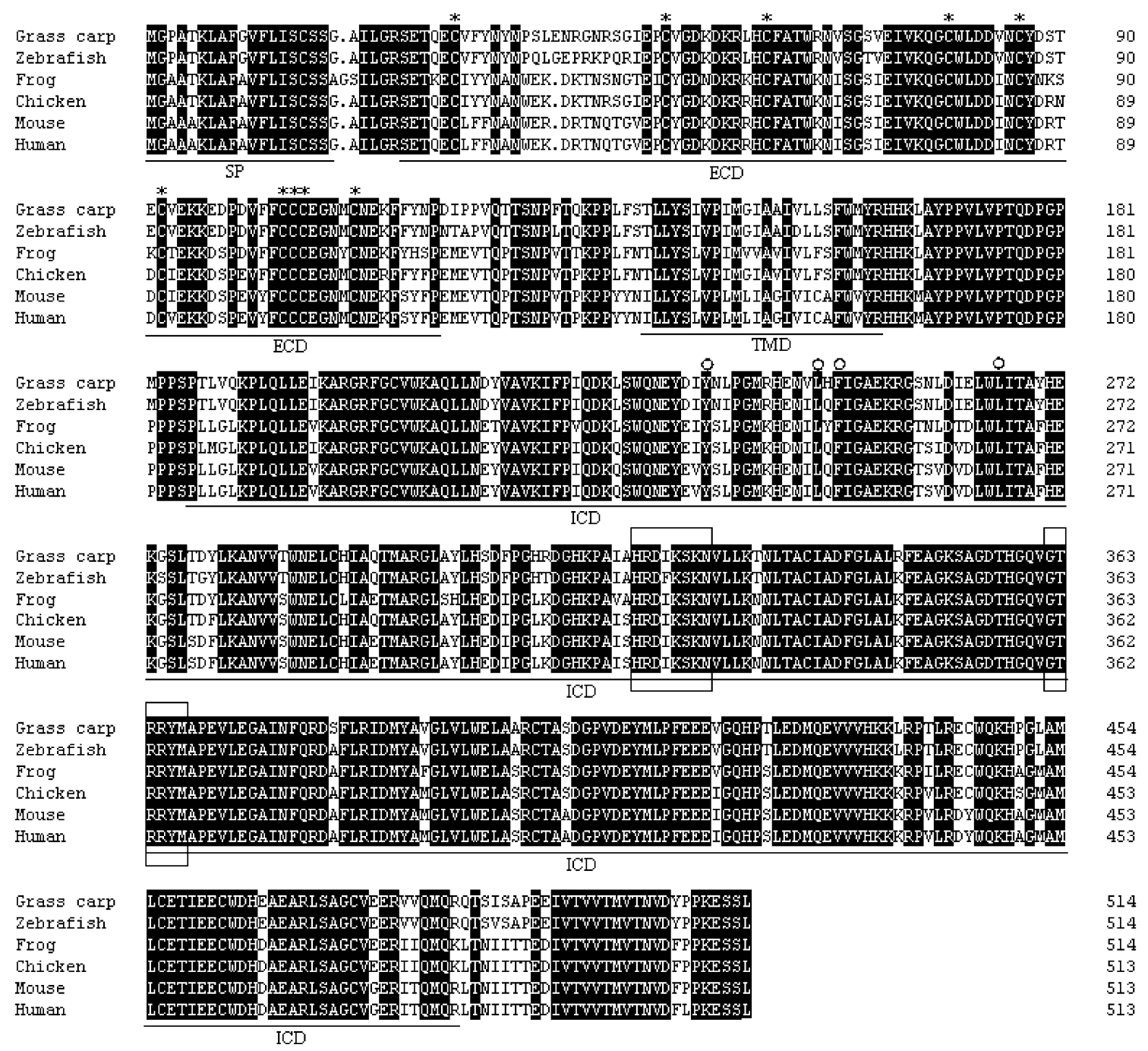

Fig. 2. Multiple alignment of deduced protein sequence of grass carp ActRIIA standard form (Variant 1) with zebrafish ActRIIA (NP_001103748), frog ActRII (NP_001084061), chicken ActRIIA (NP_990698), mouse ActRIIA (AAI38824), and human ActRIIA (AAH67417). Identical residues across species are marked by black background. Signal peptide (SP), extracellular ligand-binding domain (ECD), transmembrane domain (TMD) and intracellular serine/threonine kinase domain (ICD) are underlined separately. Ten conserved cysteines are indicated by asterisks $(*)$. Open circles indicate the residues which are involved in forming the back pocket. Two characteristic motifs of serine/threonine protein kinases are boxed. 
Protein sequence analysis of grass carp ActRIIA

The standard form of grass carp ActRIIA (variant 1) encodes a protein of 514 amino acids, which displays 7995\% identity with its orthologs from teleost, amphibian, avian and mammalian species. Amino acid sequence analysis showed that this isoform contained the characteristic regions of TGF- $\beta$ type II receptor superfamily, including a signal peptide (SP, 1-19 aa), a short extracellular domain (ECD, 25-118 aa), a hydrophobic transmembrane domain (TMD, 140-162 aa), and a large intracellular serine/ threonine kinase domain (ICD, 186485 aa). As shown in Fig. 2, in the ECD, we observed 10 conserved cysteines, which could form disulfide bonds
$\left(\mathrm{C}_{1}-\mathrm{C}_{3}, \mathrm{C}_{2}-\mathrm{C}_{4}, \mathrm{C}_{5}-\mathrm{C}_{8}, \mathrm{C}_{6}-\mathrm{C}_{7}\right.$, and $\left.\mathrm{C}_{9}-\mathrm{C}_{10}\right)$ and determine the folding of the ligand-binding domain (Greenwald et al., 1999). In the ICD, two characteristic motifs of Serine/Threonine protein kinases, HRDIKSKN (321-328 aa) and GTRRYM (362-367 aa) were identified, of which $\mathrm{D}_{323}$ was predicted to be the Serine/Threonine protein kinas active-site. $\mathrm{Y}_{237}, \mathrm{~L}_{248}, \mathrm{~F}_{250}$ and $\mathrm{L}_{266}$ are highly conserved and are predicted to form the back pocket (Fig. 2). In addition, the characteristic ESSL motif, which functions to mediate interaction between ActRIIs and PDZ-proteins (Matsuzaki et al., 2002; Tsuchida et al., 2004), was also found at the C-terminal of grass carp ActRIIA.
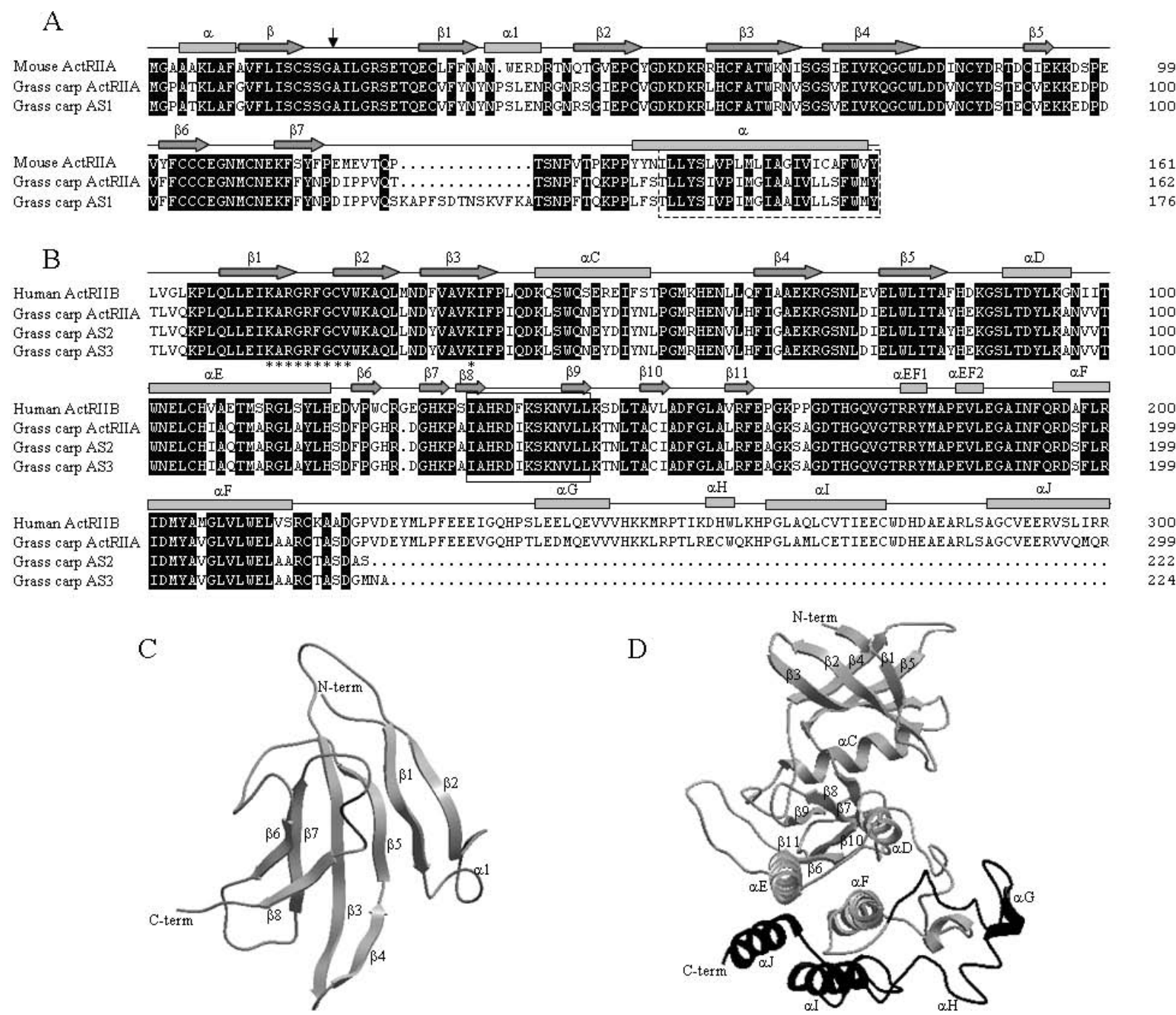

Fig. 3. (A) Secondary structure of the extracellular domain and transmembrane region of grass carp ActRIIA. The cleavage site for signal peptide is indicated by arrow. Secondary structure elements are represented by "rectangles" $(\alpha$-helices) and "arrows" ( $\beta$ strands) and structural elements in the ligand-binding domain were numbered. The transmembrane region is indicated by a dashed box. (B) Secondary structure of the intracellular kinase domain of grass carp ActRIIA. Putative ATP-binding residues are indicated by asterisks (*) and the serine/threonine kinase active-site is boxed. Secondary elements within the kinase domain were numbered. The three-dimensional structure of grass carp ActRIIA ligand-binding domain (C) and serine/threonine kinase domain (D) were constructed by comparative protein modeling program SWISS-MODEL. Truncated part of kinase domain due to AS2 and AS3 is highlighted in black. 
The secondary structure-based alignment between the standard form of grass carp ActRIIA and mouse ActRIIA showed that the ECD of grass carp ActRIIA exhibited seven $\beta$-strands, and the TMD contained one $\alpha$-helix (Fig. 3A). Also, the additional 14 amino acids derived from AS1 were located between the ECD and the TMD (Fig. 3A). When compared with human ActRIIB, the ICD of grass carp ActRIIA possessed highly conserved secondary structure elements, including a five-strand anti-parallel $\beta$-sheet and a single $\alpha$-helix $(\alpha \mathrm{C})$ in the N-terminal, and multiple $\alpha$-helices in the C-terminal (Fig. 3B). Furthermore, homology modeling studies showed that the ligandbinding domain of grass carp ActRIIA displayed the typical three-finger toxin folding (Fig. 3C and Greenwald et al., 1999). The intact kinase domain of grass carp ActRIIA exhibited the conserved bilobal architecture which was similar to all catalytic kinase domains of ActRIIA from other species (Fig. 3D), while the truncated C-terminal of the kinase domain (408-485 aa) lacked the $\alpha \mathrm{G}, \alpha \mathrm{H}, \alpha \mathrm{I}$, and $\alpha \mathrm{J}$ helix (Fig. 3B and 3D).

\section{Evolutionary analysis on type II receptors of TGF-} $\beta$ family In order to reveal the evolutionary relationship between grass carp ActRIIA and other vertebrate type II receptors of TGF- $\beta$ family, the unrooted phylogenetic tree was reconstructed on the basis of full-length protein sequences of ActRIIAs, ActRIIBs, TGFBRIIs as well as BMPRIIs. All ActRIIA proteins are grouped into one branch, distinct from ActRIIB, TGFBRII and BMPRII subfamilies (Fig. 4). Within the ActRIIA branch, grass

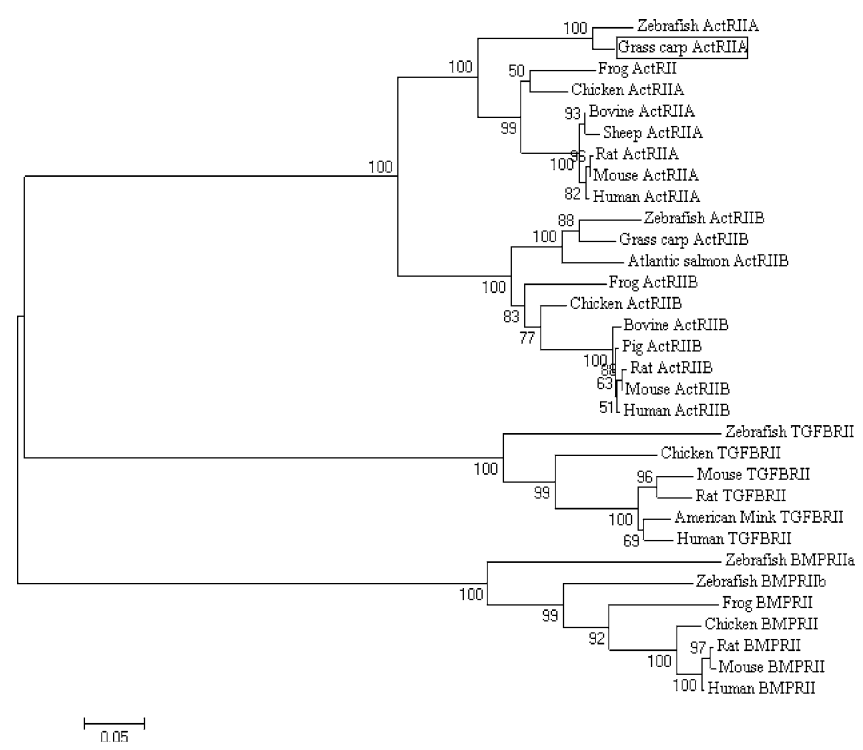

Fig. 4. Neighbor-joining guided phylogenetic tree of type II serine/threonine kinase receptors of TGF- $\beta$ family. The tree was constructed according to the alignment of complete amino acid sequences (accession nos. in the MATERIALS AND METHODS section). Bootstrap values (1000 replicates) indicating the occurrence of nodes are marked at the node of branch.

A
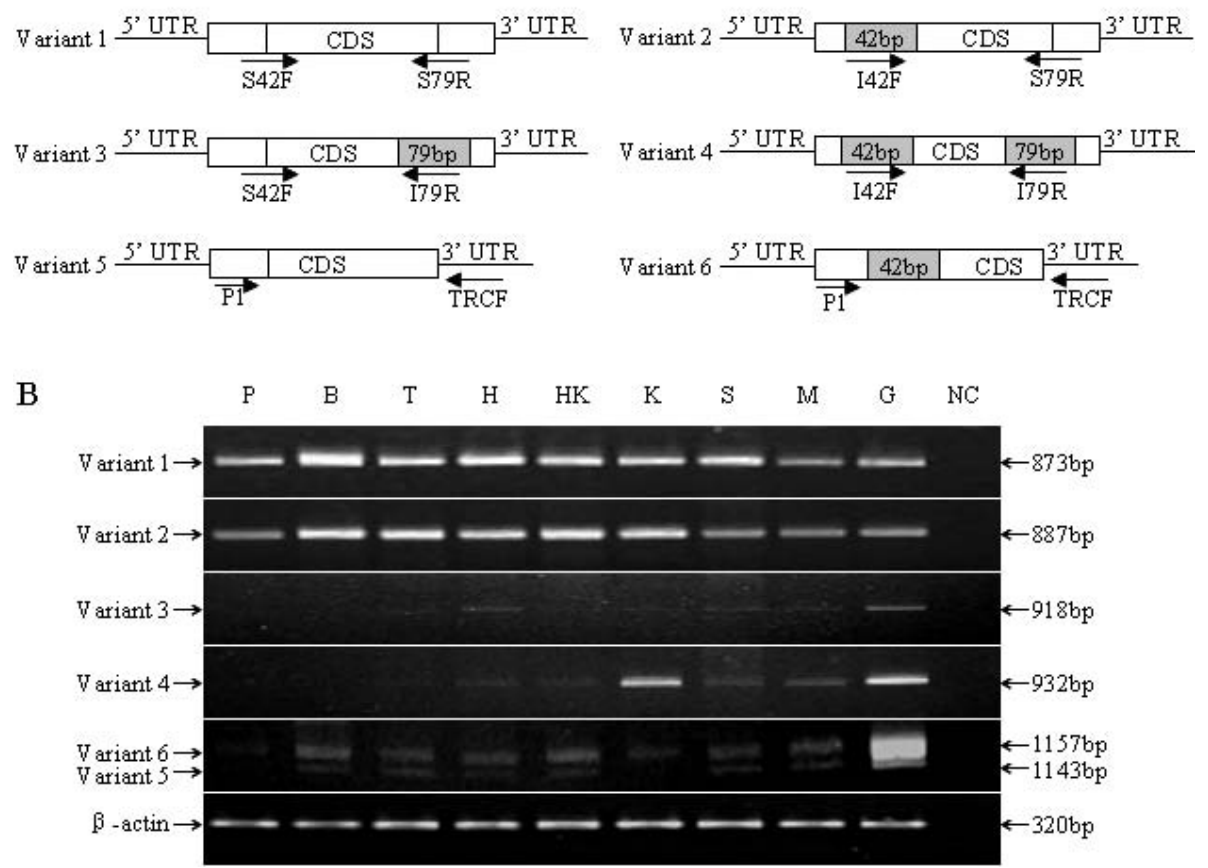

Fig. 5. (A) Schematic structures of grass carp ActRIIA splice variants and location of primers used in tissue distribution study. Putative coding sequence region (CDS) is indicated by box and alternative splicing sites are indicated by vertical lines within CDS. (B) Tissue distribution patterns of six ActRIIA variants in various tissues of grass carp by RT-PCR with $\beta$-actin as internal control. P, pituitary; B, brain; T, thymus; $\mathrm{H}$, heart; HK, head kidney; K, kidney; S, spleen; M, muscle; G, gonad; NC, negative control. 
carp ActRIIA exhibits a gradually distant evolutionary relationship with its homologs from zebrafish, frog, avian and mammals (Fig. 4).

Tissue distribution patterns of alternatively spliced variants of grass carp ActRIIA The spatial expression of grass carp ActRIIA transcript variants was examined by semi-quantitative RT-PCR using variantspecific primers (Fig. 5A). PCR products with $873 \mathrm{bp}$ (variant 1), $887 \mathrm{bp}$ (variant 2), $918 \mathrm{bp}$ (variant 3), $932 \mathrm{bp}$ (variant 4), $1171 \mathrm{bp}$ (variant 5) and $1213 \mathrm{bp}$ (variant 6) in size were consistently amplified. Both variant 1 and variant 2 were ubiquitously expressed with relatively high abundance in brain, thymus, heart, head kidney and kidney, but detected at relatively low abundance in muscle and gonad (Fig. 5B). In contrast, variant 3 was only detectable at extremely low level in a few tissues, including gonad, heart, spleen and muscle (Fig. 5B). The highest abundance of variant 4 was detected in kidney and gonad, to a lesser extent in heart, head kidney, spleen and muscle, and few copies in pituitary, brain and thymus (Fig. 5B). Variant 5 was detected in the examined tissues except for pituitary and kidney with extremely low abundance, whereas variant 6 mRNA was presented in all examined tissues, especially high expressed in gonad (Fig. 5B).

\section{DISCUSSION}

In the present study, six transcripts for ActRIIA have been cloned from grass carp. The standard form of ActRIIA (variant 1) is encoded by $1545 \mathrm{bp}$ nucleotides, and the putative ActRIIA protein shares 79-95\% identity with other vertebrate orthologs. Consistently, an early study also showed that zebrafish ActRIIA has two different transcripts in both the ovary and adult whole body of fish (Nagaso et al., 1999). In grass carp ActRIIA, an alternatively spliced (AS1) segment including 14 amino acids is located in the external juxtamembrane region of the receptor and this is consistent with findings in the mouse ActRIIA and its subfamily receptor ActRIIB. The mouse ActRIIA had a $24 \mathrm{bp}$ insertion in the juxtamembrane region, which was also identified in human and Xenopus, and the amino acid sequences of the insertion in these three species were completely conserved (Shoji et al., 1998). Also, the mouse ActRIIB has been shown to undergo alternative splicing to generate four isoforms of ActRIIB transcripts (ActRIIB1-4, Attisano et al., 1992). These isoforms differ from each other in the inclusion of an alternative spliced segment in the juxtamembrane region, and they exhibited different affinity for activin A (Attisano et al., 1992). Furthermore, de Vries et al. reported that two spliced variants of the mouse ActRIIB (1 and 4) have distinct effects on zebrafish development, and exhibit different specificities to type I receptor (de
Vries et al., 1996). Taken together, these results suggest that the alternatively spliced segments of ActRIIB located in the juxtamembrane regions are probably involved in the ligand binding affinity and the specificity of complex formation of this type II receptor with type I receptors. Recently, in vivo functions of ActRIIB splicing variants have been examined using a strain of mutant mice which produces only ActRIIB4, the shortest isoform lacking both splicing domains (Lee et al., 2006). The investigators have demonstrated that ActRIIB4 can compensate for the loss of the other three isoforms, and ActRIIB4 is capable of affecting the development of knock-in mice most likely due to reduced expression rather than the function of ActRIIB4 (Lee et al., 2006). These findings support the notion that alternative splicing has a role in regulating physiological gene expression. In this study, the ubiquitous expression of variant 1 and 2, which are two transcripts with/without AS1 respectively, has been observed (Fig. 5B). Therefore, we do not exclude the possibility that signaling of activin or other TGF family members was modulated by regulating expression level of variants with/without AS1 in different tissues/cells.

Besides AS1, we also identified other two alternative splicing events (AS2 and AS3) which occur at the same cleavage site through different splicing ways. Indeed, AS2 inserts a fragment of 79 bp nucleotides while AS3 generates a new 3' terminal of cDNA with poly (A) signals. Both AS2 and AS3 induce a premature termination codon and the encoded putative proteins differ from each other only in a few amino acid residues at the C-terminal (Fig. 1B and 3B). In this case, AS2 and AS3 created a similar truncated kinase domain which lacks four typical $\alpha$-helixes $(\alpha \mathrm{G}, \alpha \mathrm{H}, \alpha \mathrm{I}, \alpha \mathrm{J})$ at the C-terminal lobe (Fig. 3B). In ActRII family, to our knowledge, only the crystal structure of human ActRIIB kinase domain has been solved, revealing the existence of the conserved "gatekeeper residues" which play an important structural role in active site (Han et al., 2007). Interestingly, multiple sequence alignment showed that all of "gatekeeper residues" are highly conserved in grass carp ActRIIA variant $1\left(\mathrm{Y}_{237}, \mathrm{~L}_{248}, \mathrm{~F}_{250}, \mathrm{~L}_{266}\right.$, see Fig. 2). It has been reported that when the truncated activin type II receptor lacking the cytoplasmic kinase domain was expressed in K562 cells, the induction of erythroid differentiation by activin was abrogated in these cells, indicating that the truncated receptor could bind activin but was not capable of transmitting activin signals because of the impaired kinase domain (Liu et al., 2000). Therefore, we speculated that grass carp ActRIIA might mediate the activin signaling through selectively expressing transcripts with the intact and truncated kinase domain. In addition, recent studies have revealed that up to one-third of human alternative splicing events create an in-frame premature termination codon (PTC) that would result in non- 
sense-mediated mRNA decay (NMD) (McGlincy and Smith, 2008). In this study, we cannot eliminate the possibility that AS2 and AS3, which both contain a PTC, would cause the resulting grass carp ActRIIA mRNA to be degraded by NMD. Taken together, it is possible that alternative splicing of grass carp ActRIIA mRNA not only generates protein diversity, but also regulates transcript abundance, although the functional roles of these variants need to be investigated.

Tissue distribution assay has confirmed the existence of AS1, AS2 and AS3 in grass carp. It is worth mentioning that steady-state mRNA levels of six variants were tissuespecific in this species. For example, variant 1 and 2 showed wider tissue expression pattern than other four variants, and these two variants may be the predominant isoforms in grass carp since they were detected in all examined tissues with relatively high abundance. In addition, those variants induced by AS2 and AS3 (variant $3,4,5$ and 6) were detected to be predominantly expressed in the gonad, implying the potential role of these variants in the reproductive axis. Interestingly, ActRIIA transcripts were detected with the highest level in the oocyte during zebrafish embryonic development (Nagaso et al., 1999). These findings indicated that alternative splicing could potentially introduce additional complexity to the activity of activin and other members of TGF- $\beta$ family in grass carp.

In summary, we have originally identified that six splice variants can be generated through alternative splicing in the grass carp ActRIIA mRNA. To our knowledge, no such types of alternative splicing in the ActRIIA mRNA have so far been described in lower vertebrates. The tissue distribution patterns showed that these variants were ubiquitously expressed in grass carp, suggesting the functional importance of these splice variants. Although it is well documented that TGF- $\beta$ ligands exhibit a diversity of physiological roles through sharing a few receptors, the mechanism how these receptors can fine bind to ligands and tune multiple signals is unknown. Our findings reveal the presence of novel variants of ActRII in teleost, indicating that alternative splicing might be an important way to signaling regulation at the receptor level in the TGF- $\beta$ superfamily.

This work was sponsored by grants from National Natural Science Foundation of China (30650003) and Program for New Century Excellent Talents in University (NCEF-06-0814) to H. Z.

\section{REFERENCES}

Altschul, S. F., Gish, W., Miller, W., Myers, E. W., and Lipman, D. J. (1990) Basic local alignment search tool. J. Mol. Biol. 215, 403-410.

Attisano, L., Carcamo, J., Ventura, F., Weis, F. M., Massague, J., and Wrana, J. L. (1993) Identification of human activin and TGF beta type I receptors that form heteromeric kinase complexes with type II receptors. Cell 75, 671-680.

Attisano, L., Wrana, J. L., Cheifetz, S., and Massague, J. (1992) Novel activin receptors: distinct genes and alternative mRNA splicing generate a repertoire of serine/threonine kinase receptors. Cell 68, 97-108.

Bendtsen, J. D., Nielsen, H., von Heijne, G., and Brunak, S. (2004) Improved prediction of signal peptides: SignalP 3.0. J. Mol. Biol. 340, 783-795.

Chang, H., Brown, C. W., and Matzuk, M. M. (2002) Genetic analysis of the mammalian transforming growth factor-beta superfamily. Endocr. Rev. 23, 787-823.

de Vries, C. J., de Boer, J., Joore, J., Strahle, U., van Achterberg, T. A., Huylebroeck, D., Verschueren, K., Miyazono, K., van den Eijinden-van Raaij, A. J., and Zivkovic, D. (1996) Active complex formation of type I and type II activin and TGF beta receptors in vivo as studied by overexpression in zebrafish embryos. Mech. Dev. 54, 225-236.

DiMuccio, T., Mukai, S. T., Clelland, E., Kohli, G., Cuartero, M., $\mathrm{Wu}$, T., and Peng, C. (2005) Cloning of a second form of activin-betaA cDNA and regulation of activin-betaA subunits and activin type II receptor mRNA expression by gonadotropin in the zebrafish ovary. Gen. Comp. Endocrinol. 143, 287-299.

Donaldson, C. J., Mathews, L. S., and Vale, W. W. (1992) Molecular cloning and binding properties of the human type II activin receptor. Biochem. Biophys. Res. Commun. 184, 310-316.

Falquet, L., Pagni, M., Bucher, P., Hulo, N., Sigrist, C. J., Hofmann, K., and Bairoch, A. (2002) The PROSITE database, its status in 2002. Nucleic Acids Res. 30, 235-238.

Greenwald, J., Fischer, W. H., Vale, W. W., and Choe, S. (1999) Three-finger toxin fold for the extracellular ligand-binding domain of the type II activin receptor serine kinase. Nat. Struct. Biol. 6, 18-22.

Han, S., Loulakis, P., Griffor, M., and Xie, Z. (2007) Crystal structure of activin receptor type IIB kinase domain from human at 2.0 Angstrom resolution. Protein Sci. 16, 22722277.

Jones, K. L., Mansell, A., Patella, S., Scott, B. J., Hedger, M. P., de Kretser, D. M., and Phillips, D. J. (2007) Activin A is a critical component of the inflammatory response, and its binding protein, follistatin, reduces mortality in endotoxemia. Proc. Natl. Acad. Sci. USA 104, 1623916244.

Kumar, S., Tamura, K., and Nei, M. (2004) MEGA3: Integrated software for Molecular Evolutionary Genetics Analysis and sequence alignment. Brief Bioinform. 5, 150-163.

Lavery, K., Swain, P., Falb, D., and Alaoui-Ismaili, M. H. (2008) BMP-2/4 and BMP-6/7 differentially utilize cell surface receptors to induce osteoblastic differentiation of human bone marrow-derived mesenchymal stem cells. J. Biol. Chem. 283, 20948-20958.

Lee, Y. J., Hong, K. H., Yun, J., and Oh, S. P. (2006) Generation of activin receptor type IIB isoform-specific hypomorphic alleles. Genesis 44, 487-494.

Ling, N., Ying, S. Y., Ueno, N., Shimasaki, S., Esch, F., Hotta, M., and Guillemin, R. (1986) Pituitary FSH is released by a heterodimer of the beta-subunits from the two forms of inhibin. Nature 321, 779-782.

Liu, F., Shao, L. E., and Yu, J. (2000) Truncated activin type II receptor inhibits erythroid differentiation in K562 cells. J. Cell. Biochem. 78, 24-33.

Matsuzaki, T., Hanai, S., Kishi, H., Liu, Z., Bao, Y., Kikuchi, A. Tsuchida, K., and Sugino, H. (2002) Regulation of endocytosis of activin type II receptors by a novel PDZ protein 
through Ral/Ral-binding protein 1-dependent pathway. J. Biol. Chem. 277, 19008-19018.

McGlincy, N. J., and Smith, C. W. (2008) Alternative splicing resulting in nonsense-mediated mRNA decay: what is the meaning of nonsense? Trends Biochem. Sci. 33, 385-393.

Mizuguchi, T., Kosaka, M., and Saito, S. (1993) Activin A suppresses proliferation of interleukin-3-responsive granulocyte-macrophage colony-forming progenitors and stimulates proliferation and differentiation of interleukin-3-responsive erythroid burst-forming progenitors in the peripheral blood. Blood 81, 2891-2897.

Nagaso, H., Suzuki, A., Tada, M., and Ueno, N. (1999) Dual specificity of activin type II receptor ActRIIb in dorsoventral patterning during zebrafish embryogenesis. Develop. Growth Differ. 41, 119-133.

Robson, N. C., Phillips, D. J., McAlpine, T., Shin, A., Svobodova, S., Toy, T., Pillay, V., Kirkpatrick, N., Zanker, D., Wilson, K., et al. (2008) Activin-A: a novel dendritic cell-derived cytokine that potently attenuates CD40 ligand-specific cytokine and chemokine production. Blood 111, 27332743.

Shi, Y., and Massague, J. (2003) Mechanisms of TGF-beta signaling from cell membrane to the nucleus. Cell 113, 685700 .

Shoji, H., Nakamura, T., van den Eijnden-van Raaij, A. J., and Sugino, H. (1998) Identification of a novel type II activin receptor, type IIA-N, induced during the neural differentiation of murine P19 embryonal carcinoma cells. Biochem. Biophys. Res. Commun. 246, 320-324.

Tsuchida, K., Nakatani, M., Matsuzaki, T., Yamakawa, N., Liu, Z., Bao, Y., Arai, K. Y., Murakami, T., Takehara, Y., Kurisaki, A., and Sugino, H. (2004) Novel factors in regulation of activin signaling. Mol. Cell. Endocrinol. 225, 1-8.

Vale, W., Rivier, J., Vaughan, J., McClintock, R., Corrigan, A., Woo, W., Karr, D., and Spiess, J. (1986) Purification and characterization of an FSH releasing protein from porcine ovarian follicular fluid. Nature 321, 776-779.

Wang, Q. F., Tilly, K. I., Tilly, J. L., Preffer, F., Schneyer, A. L., Crowley, W. F. Jr., and Sluss, P. M. (1996) Activin inhibits basal and androgen-stimulated proliferation and induces apoptosis in the human prostatic cancer cell line, LNCaP. Endocrinology 137, 5476-5483.

Wang, X. P., Suomalainen, M., Felszeghy, S., Zelarayan, L. C., Alonso, M. T., Plikus, M. V., Maas, R. L., Chuong, C. M., Schimmang, T., and Thesleff, I. (2007) An integrated gene regulatory network controls stem cell proliferation in teeth. PLoS Biol. 5, e159.

Xiao, L., Yuan, X., and Sharkis, S. J. (2006) Activin A maintains self-renewal and regulates fibroblast growth factor, Wnt, and bone morphogenic protein pathways in human embryonic stem cells. Stem Cells 24, 1476-1486. 